\title{
BMJ Open Relationship between diagonal earlobe creases and coronary artery disease as determined via angiography
}

\author{
Yong Wang, ${ }^{1,2}$ Li-Hua Mao, ${ }^{1,3}$ En-Zhi Jia, ${ }^{1}$ Zhao-Yang Li, ${ }^{1}$ Xiao-Qing Ding, ${ }^{1}$ \\ Peng-Cheng Ge, ${ }^{1}$ Zhe Liu, ${ }^{1}$ Tie-Bing Zhu, ${ }^{1}$ Lian-Sheng Wang, ${ }^{1}$ Chun-Jian Li, ${ }^{1}$ \\ Wen-Zhu Ma, ${ }^{1}$ Zhi-Jian Yang ${ }^{1}$
}

To cite: Wang Y, Mao L-H, Jia E-Z, et al. Relationship between diagonal earlobe creases and coronary artery disease as determined via angiography. BMJ Open 2016;6:e08558.

doi:10.1136/bmjopen-2015008558

- Prepublication history for this paper is available online. To view these files please visit the journal online (http://dx.doi.org/10.1136/ bmjopen-2015-008558)

Yong Wang and Li-Hua Mao contributed equally to this work.

Received 23 April 2015 Revised 2 December 2015 Accepted 4 January 2016

CrossMark

For numbered affiliations see end of article.

Correspondence to

Dr En-Zhi Jia;

enzhijiacn@aliyun.com

\section{ABSTRACT}

Objective: This study was designed to examine the prevalence of unilateral and bilateral diagonal earlobe creases (DELCs) with respect to the diagnosis of coronary heart disease (CHD).

Methods: A total of 558 consecutive participants (402 males and 156 females) aged 36-91 years who underwent coronary angiography were enrolled in this study. The participants were classified as being without a DELC, having a unilateral DELC and having bilateral DELCs; participants with either a unilateral DELC or bilateral DELCs were defined as participants with DELCs. Significant CHD was defined as at least one major vessel with $>50 \%$ stenosis, and coronary atherosclerosis severity was defined using the Gensini scoring system.

Results: In the present study, bilateral DELCs were more frequently among male $(p=0.001), C H D$ $(p=0.000)$, older people $(p=0.000)$ and those with more severe coronary artery atherosclerosis $(p=0.000)$. The results of the multiple regression analyses indicated that DELCs $(\mathrm{OR}, 4.861 ; 95 \% \mathrm{Cl} 3.093$ to $7.642, p=0.000$ ) remained independently associated with a risk of CHD. It was assumed that participants without a DELC have a certain background risk for CHD ( $O R$ is assumed to be 1); the results of the multivariate logistic regression indicated that the relative risk of CHD among participants with bilateral DELCs was 5.690 among all participants (OR, 5.690; $95 \% \mathrm{Cl}$ 3.450 to $9.384, p=0.000), 5.436$ among male participants (OR, $5.436 ; 95 \% \mathrm{Cl} 2.808$ to 10.523 , $\mathrm{p}=0.000$ ) and 7.148 among female participants ( $0 \mathrm{R}$, 7.148; $95 \% \mathrm{Cl} 3.184$ to $16.049, p=0.000$ ). Moreover, a positive association between DELC and age $(\mathrm{SI}=1.21$, $\mathrm{SIM}=1.65, \mathrm{AP}=0.132)$, gender $(\mathrm{SI}=2.09, \mathrm{SIM}=0.81$, $\mathrm{AP}=0.49)$ and smoking status $(\mathrm{SI}=1.49, \mathrm{SIM}=0.73$, $A P=0.29$ ) was found, respectively.

Conclusions: The results of the present study indicated that DELCs are a simple and a feasible means of identifying CHD. However, the exact mechanism underlying the relationship between DELCs and CHD warrants further study.

\section{INTRODUCTION}

Coronary heart disease (CHD) is the leading cause of death worldwide; its prevention is a

\section{Strengths and limitations of this study}

- The strength of this study is that it used coronary angiography and a standard evaluation sheet for the diagnosis of coronary heart disease (CHD) and diagonal earlobe creases (DELC), respectively.

- Further, the study explored the differentiation of unilateral versus bilateral DELCs for the diagnosis of CHD. However, its sample size was small; and the pathogenesis of the apparent relationship between DELC and CHD was not studied.

- Although a statistical relationship appears to exist, the biological plausibility of said relationship remains unproven and warrants clarification in future studies.

public health priority. ${ }^{1}$ As more than 1.3 billion people live in China, the morbidity and mortality of CHD has been growing within the past 30 years. To decrease the burden imposed by the disease, ${ }^{2}$ the exploration of the usefulness of various simple and reliable signs of atherosclerosis with respect to the identification of the participants at risk for CHD is urgently needed. ${ }^{3}$ Preliminary observations by ancient Chinese traditional doctors suggesting that a 'positive ear-lobe sign' is associated with the development of premature coronary artery atherosclerosis have been heralded. ${ }^{4}$ The diagonal earlobe crease (DELC) is the fold or crease in the skin of the ear-lobe originally described by Frank in $1973 .{ }^{5}$ Several other reports regarding DELC as a risk factor for CHD have since been published. ${ }^{6}$ Although many studies regarding the relationship between DELC and CHD have been undertaken, very few have focused on differences regarding the correlation of unilateral or bilateral DELCs with CHD. Cohort studies suggest that high-risk participants with unilateral, bilateral, or no earlobe creases, have 
different prognoses of CHD. ${ }^{78}$ This study was designed to study the prevalence of unilateral and bilateral DELCs with respect to the prevalence of CHD.

\section{PARTICIPANTS AND METHODS \\ Study participants}

From May 2014 to October 2014, 558 consecutive participants (402 males and 156 females) aged 36-91 years, who underwent coronary angiography for either suspected or known coronary atherosclerosis at the First Affiliated Hospital of Nanjing Medical University, were enrolled in this study. The exclusion criteria were as follows: participants suffering from spastic angina pectoris, heart failure, infectious processes within 2 weeks of enrolment, adrenal dysfunction or thyroid dysfunction. This study was approved by the ethics committee of the First Affiliated Hospital of Nanjing Medical University. All participants gave written informed consent.

\section{Coronary angiography}

The coronary arteries were cannulated using either the Judkins technique ${ }^{9}$ or a radial approach, using $6 \mathrm{~F}$ catheters, and recorded at a rate of 30 frames/s. The presence of coronary artery stenosis was evaluated following the intracoronary injection of isosorbide dinitrate (ISDN; $2.5 \mathrm{mg} / 5 \mathrm{~mL}$ solution over $20 \mathrm{~s}$ ). Significant CHD was defined as at least one major epicardial vessel with $>50 \%$ stenosis, whereas the control was defined as $<50 \%$ stenosis within each of the major epicardial vessels. ${ }^{10}$ Coronary atherosclerosis severity was defined using the Gensini scoring system. The Gensini score was computed by assigning a severity score to the area of each coronary stenosis according to the degree of luminal narrowing and the lesion's geographic importance. ${ }^{11}$

\section{DELC criteria}

A modified evaluation sheet designed by Isha Shrestha was used to assess the DELCs, with the participants seated. ${ }^{12}$ A deep diagonal crease $(>1 \mathrm{~mm})$ extending obliquely from the tragus towards the outer border of the ear lobe and covering at least two-thirds of the length of the ear lobe was recorded as a DELC. Two trained observers blinded to the participants' diagnoses were employed to examine the participants' DELC statuses and had consensus regarding the presence or absence of a DELC. Subjects' DELC statuses were classified as being without a DELC (figures 1 and 2), having a unilateral DELC (figures 3 and 4) and having bilateral DELCs (figures 5 and 6), and participants with either a unilateral DELC or bilateral DELCs were defined as participants with DELCs.

\section{Cigarette smoking}

Cigarette smoking was assessed using a standardised questionnaire. The participants' smoking status was

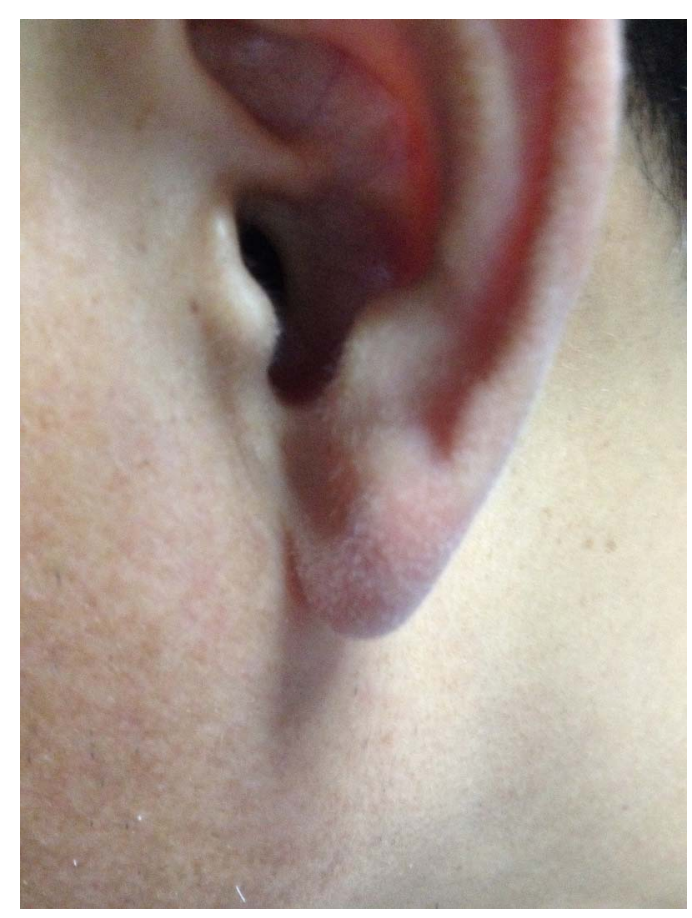

Figure 1 The participants' DELC status was classified as without DELC (left). DELC, diagonal earlobe crease.

classified as either 'not smoking' or 'smoking' (the latter group included both former and current smokers).

\section{Blood pressure measurement}

Systolic and diastolic blood pressures were measured by a manual sphygmomanometer in a sitting position after resting for $10 \mathrm{~min}$ and three recordings were gathered for each subject (2 min interval between successive

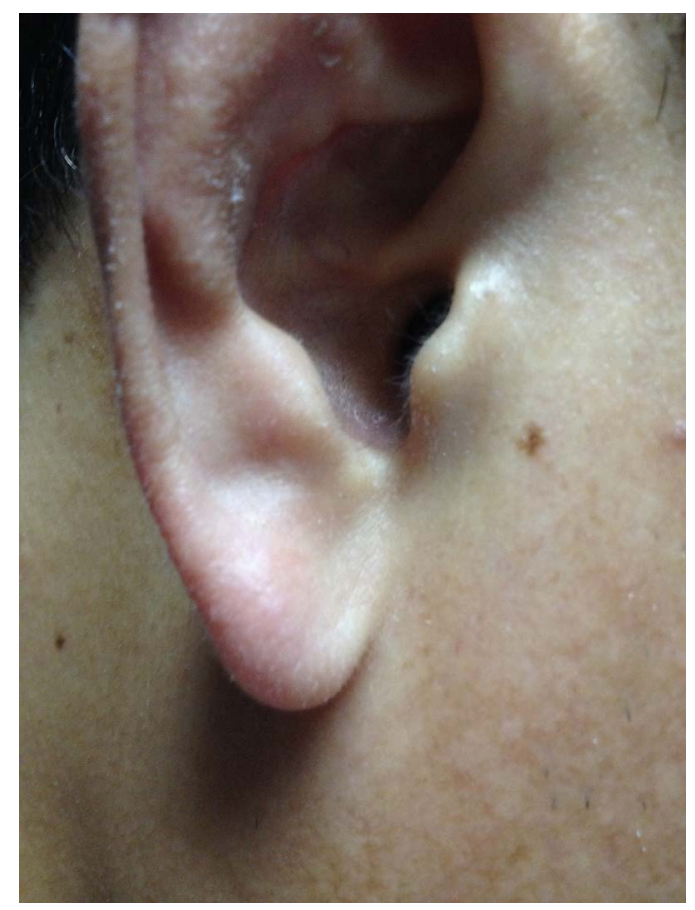

Figure 2 The participants' DELC status was classified as without DELC (right). DELC, diagonal earlobe crease. 


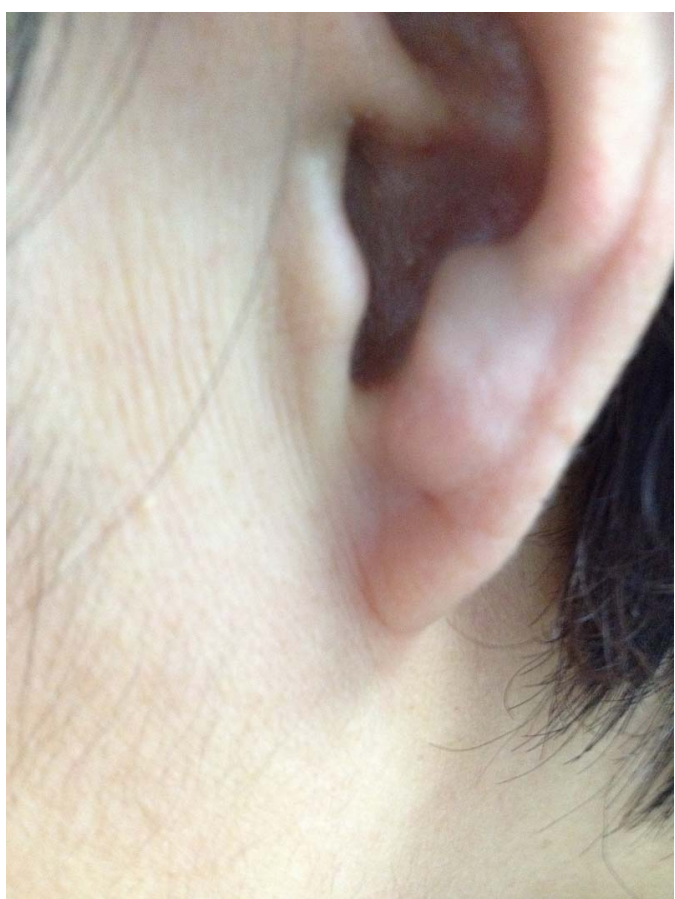

Figure 3 The participants' DELC status was classified as unilateral DELC (left). DELC, diagonal earlobe crease.

measurements). The mean systolic ( $\mathrm{SBP}, \mathrm{mm} \mathrm{Hg}$ ) and diastolic blood pressures (DBP, $\mathrm{mm} \mathrm{Hg}$ ) were recorded.

\section{Laboratory measurements}

Total cholesterol (TCH, mmol/L), triglycerides (TG, $\mathrm{mmol} / \mathrm{L}$ ), fasting blood glucose (FBG, $\mathrm{mmol} / \mathrm{L}$ ), fasting high-density lipoprotein cholesterol (HDL-c, mmol/L)

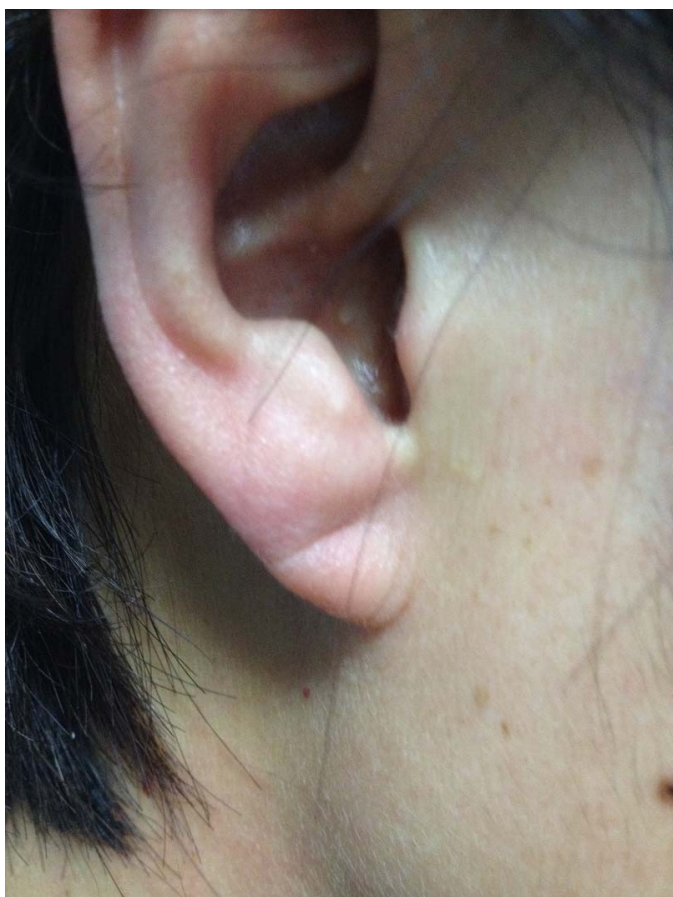

Figure 4 The participants' DELC status was classified as unilateral DELC (right). DELC, diagonal earlobe crease.

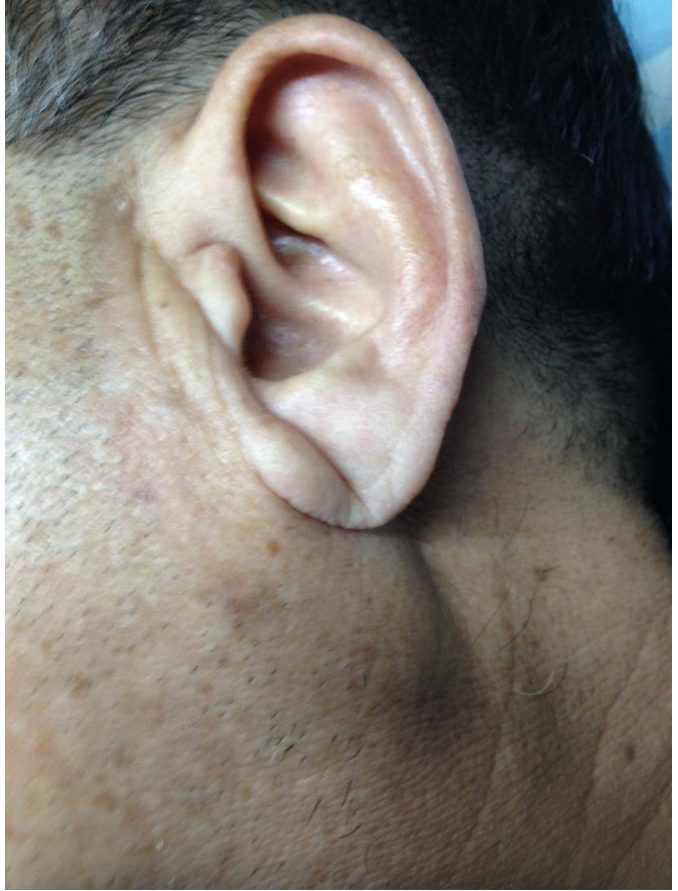

Figure 5 The participants' DELC status was classified as bilateral DELC (left). DELC, diagonal earlobe crease.

and fasting low-density lipoprotein cholesterol (LDL-c, $\mathrm{mmol} / \mathrm{L}$ ), were measured via enzymatic procedures using an automated autoanalyser (AU 2700 Olympus, 1st Chemical Ltd, Japan).

\section{Statistical analysis}

The data were analysed using the Statistics Package for Social Sciences (V.16.0; SPSS Incorporated, Chicago,

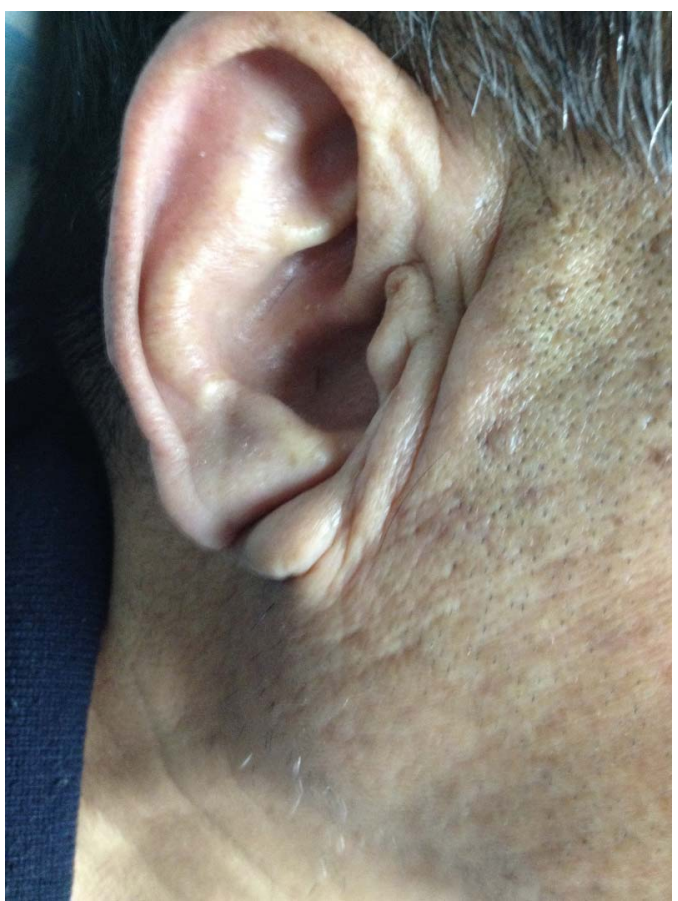

Figure 6 The participants' DELC status was classified as bilateral DELC (right). DELC, diagonal earlobe crease. 
Illinois, USA). The participants were classified into 2 or 3 groups according to their CHD or DELC statuses. Data regarding age and LDL-c were normally distributed parameters and were therefore presented as means \pm SDs; comparisons were analysed via either an independentsamples $\mathrm{t}$ test or a one-way analysis of variance (ANOVA). Skewed data, including TCH, HDL-c, TG, FBG and Gensini scores were expressed as medians and IQRs, and comparisons were analysed via either the Mann-Whitney $\mathrm{U}$ test or the Kruskal-Wallis test. Categorical variables such as gender, smoking status, CHD status and DELC status, were compared between the groups, using a $\chi^{2}$ analysis. ORs regarding the presence of obstructive CHD were determined via either a univariate or a multivariate logistic regression analysis and are presented with 95\% CIs.

To analyse possible positive or negative interactions among DELC and age and gender, a $4 \times 2$ table was used to calculate ORs, $95 \%$ CIs and two-tailed $p$ values, as well as synergy measures either in additive (SI) or multiplicative models (SIM). It was assumed that unexposed individuals without a DELC have a certain background risk for CHD (OR00 is assumed to be 1); OR10 referred to the relative risk of CHD among patients without a DELC for CHD who were exposed to environmental risk factors compared with those without a DELC or exposure; OR01 referred to the relative risk among people with a DELC who were not exposed to the risk factor relative to those without a DELC or exposure; OR11 was the ratio of CHD risk among exposed patients with a DELC to the CHD risk among unexposed patients without a DELC. These ORs were participants used in the calculation of the synergy indexes: $\mathrm{SI}=(\mathrm{OR} 11-1) /(\mathrm{OR} 10+\mathrm{OR} 01-2)$; $\mathrm{SIM}=\mathrm{OR} 11 /(\mathrm{OR} 10 \times \mathrm{OR} 01)$, the relative excess risk due to interaction, RERI=OR11-OR10-OR01+1 and the attributable proportion of the disease due to the interaction, AP=RERI/OR11. ${ }^{13}$ Differences were considered significant if the null hypothesis could be rejected with $>95 \%$ confidence. All $\mathrm{p}$ values are two tailed.
The sensitivity, specificity and predictive value of the DELCs were computed, and the area under the receiver operating characteristic (ROC) curve was computed. ${ }^{14}$

\section{RESULTS}

The baseline characteristics of the participants

A total of 558 participants (402 males and 156 females) with a mean age of 63.63 years were enrolled in this study. The demographic and clinical characteristics of the participants are presented in tables 1-3. There were significant differences between the with DELC and without-DELC participants with respect to age $(p=0.000)$, sex $(p=0.001)$, SBP $(p=0.009)$, CHD status $(p=0.000)$ and Gensini scores $(p=0.000)$; there were also significant differences between the with $\mathrm{CHD}$ and without-CHD participants with respect to age $(p=0.046)$, sex $(p=0.000)$, smoking status $(p=0.002)$, glucose $(p=0.027)$, SBP $(p=0.045)$ and DELC status $(p=0.000)$. Moreover, significant differences among the unilateral DELC, bilateral DELCs and without-DELC participants with respect to age $(p=0.000)$, sex $(p=0.001)$, SBP $(\mathrm{p}=0.018)$, CHD status $(\mathrm{p}=0.000)$ and Gensini scores $(\mathrm{p}=0.000)$ were noted in this population.

\section{The univariate logistic regression analysis of the characteristics of the CHD and non-CHD groups}

The results of the univariate logistic regression analysis of the characteristics of the CHD and non-CHD groups are included in table 4. A 1-year increase in age was associated with a $2.0 \%$ increased risk of CHD (OR, 1.020; 95\% CI 1.000 to $1.041, \mathrm{p}=0.047$ ) in all participants, and a $7.3 \%$ increased risk of CHD (OR, 1.073; 95\% CI 1.029 to $1.119, \mathrm{p}=0.001)$ among female participants. Compared with male participants, the risk for CHD among female participants decreased by $72 \%$ (OR, $0.281 ; 95 \%$ CI 0.183 to $0.433, p=0.000$ ). Additionally, smokers were $109.4 \%$ more likely to suffer from CHD (OR, 2.094; 95\% CI 1.291 to 3.398, $\mathrm{p}=0.003$ ) compared

\begin{tabular}{|c|c|c|c|c|c|}
\hline Characteristic & $\begin{array}{l}\text { With a unilateral } \\
\text { DELC }(n=91)\end{array}$ & $\begin{array}{l}\text { With bilateral DELCs } \\
(\mathrm{n}=298)\end{array}$ & $\begin{array}{l}\text { Without a DELC } \\
(n=169)\end{array}$ & $\begin{array}{l}\text { Statistical } \\
\text { parameter }\end{array}$ & p Value \\
\hline Age (years) & $62.69 \pm 9.21$ & $66.88 \pm 9.98$ & $58.41 \pm 9.55$ & 41.392 & 0.000 \\
\hline Sex (male/female) & $74 / 17$ & $223 / 75$ & $105 / 64$ & 13.284 & 0.001 \\
\hline Smoking status (yes/no) & $39 / 52$ & $100 / 198$ & $52 / 117$ & 3.967 & 0.138 \\
\hline Total cholesterol $(\mathrm{mmol} / \mathrm{L})$ & 4.13 (3.49 to 4.89 ) & 4.02 (3.39 to 4.84$)$ & 4.08 (3.39 to 4.79 ) & 0.349 & 0.840 \\
\hline Triglycerides (mmol/L) & 1.50 (1.20 to 2.15$)$ & 1.51 (1.11 to 2.09$)$ & $1.39(1.04$ to 2.11$)$ & 2.826 & 0.243 \\
\hline Glucose (mmol/L) & 5.34 (4.81 to 6.19$)$ & 5.27 (4.72 to 6.24$)$ & 5.17 (4.78 to 5.93$)$ & 1.156 & 0.561 \\
\hline HDL-c (mmol/L) & 0.99 (0.88 to 1.17$)$ & $1.00(0.84$ to 1.17$)$ & $1.01(0.88$ to 1.19$)$ & 1.298 & 0.523 \\
\hline LDL-c (mmol/L) & $2.63 \pm 0.82$ & $2.59 \pm 0.82$ & $2.55 \pm 0.78$ & 0.315 & 0.730 \\
\hline $\mathrm{SBP}(\mathrm{mm} \mathrm{Hg})$ & 128 (120 to 145$)$ & 130 (120 to 144$)$ & 127 (120 to 138$)$ & 8.020 & 0.018 \\
\hline $\mathrm{DBP}(\mathrm{mm} \mathrm{Hg})$ & 75 (70 to 82$)$ & 75 (70 to 84$)$ & 78 (70 to 81$)$ & 0.970 & 0.616 \\
\hline CHD (yes/no) & $77 / 14$ & $268 / 30$ & $100 / 69$ & 64.781 & 0.000 \\
\hline Gensini scores & 31.00 (8.00 to 57.00$)$ & 35.00 (16.00 to 68.00$)$ & 10.00 (2.00 to 33.50$)$ & 54.099 & 0.000 \\
\hline
\end{tabular}

$\mathrm{CHD}$, coronary heart disease; DBP, diastolic blood pressure; DELC, diagonal earlobe crease; HDL-c, fasting high-density lipoprotein cholesterol; LDL-c, fasting low-density lipoprotein cholesterol; SBP, systolic blood pressure. 
Table 2 Characteristics of the participants with and without DELCs

\begin{tabular}{|c|c|c|c|c|}
\hline Characteristic & With DELC $(n=389)$ & Without DELC $(n=169)$ & T or $Z$ or $\chi^{2}$ test & p Value \\
\hline Age (years) & $65.89 \pm 9.95$ & $58.41 \pm 9.55$ & -8.271 & 0.000 \\
\hline Sex (male/female) & $297 / 92$ & $105 / 64$ & 11.827 & 0.001 \\
\hline Smoking status (yes/no) & $139 / 250$ & $52 / 117$ & 1.289 & 0.256 \\
\hline Total cholesterol (mmol/L) & 4.05 (3.43 to 4.85$)$ & 4.08 (3.39 to 4.79 ) & -0.314 & 0.754 \\
\hline Triglycerides (mmol/L) & 1.51 (1.13 to 2.11$)$ & 1.39 (1.04 to 2.11$)$ & -1.648 & 0.099 \\
\hline Glucose (mmol/L) & 5.27 (4.74 to 6.24$)$ & 5.17 (4.78 to 5.93$)$ & -0.875 & 0.382 \\
\hline HDL-c (mmol/L) & 0.99 (0.85 to 1.17$)$ & 1.01 (0.88 to 1.19$)$ & -1.103 & 0.270 \\
\hline LDL-c (mmol/L) & $2.61 \pm 0.81$ & $2.55 \pm 0.78$ & -0.730 & 0.466 \\
\hline $\mathrm{SBP}(\mathrm{mm} \mathrm{Hg})$ & $130(120$ to 144$)$ & 127 (120 to 138$)$ & -2.600 & 0.009 \\
\hline $\mathrm{DBP}(\mathrm{mm} \mathrm{Hg})$ & 75 (70 to 84$)$ & $78(70$ to 81$)$ & -0.580 & 0.562 \\
\hline CHD (yes/no) & $345 / 44$ & $100 / 69$ & 63.560 & 0.000 \\
\hline Gensini scores & 34.00 (14.25 to 65.00$)$ & 10.00 (2.00 to 33.50$)$ & -7.273 & 0.000 \\
\hline
\end{tabular}

with non-smokers. Moreover, compared with the participants without DELCs, the participants with DELCs were $441.0 \%$ more likely to suffer from CHD (OR, 5.410; 95\% CI 3.489 to $8.390, \mathrm{p}=0.000)$; among the entire population, male participants were $355.8 \%$ more likely to suffer from CHD (OR, 4.558; 95\% CI 2.536 to 8.191, $\mathrm{p}=0.000)$ and female participants were $461.5 \%$ more likely to suffer from CHD (OR, 5.615; 95\% CI 2.762 to 11.418, $\mathrm{p}=0.000$ ).

The risk of CHD increased significantly among the participants with a unilateral DELC compared with the participants without DELCs among the entire population, and among males and females; the ORs and 95\% CIs were as follows: $3.795,1.987$ to 7.246 , and $\mathrm{p}<0.001$ for all participants with a unilateral DELC; 3.026, 1.341 to 6.825 , and $\mathrm{p}=0.008$ for the male participants with a unilateral DELC; $3.508,1.104$ to 11.149 , and $p=0.033$ for the female participants with a unilateral DELC. Additionally, the participants with bilateral DELCs were $516.4 \%$ more likely to suffer from CHD (OR, 6.164; $95 \%$ CI 3.790 to $10.024, \mathrm{p}=0.000$ ) compared with the participants without DELCs. Moreover, the male participants with bilateral DELCs were $442.0 \%$ more likely to suffer from CHD (OR, 5.420; 95\% CI 2.804 to 10.477, $\mathrm{p}=0.000$ ) compared with the participants without DELCs; the female participants with bilateral DELCs were $536.8 \%$ more likely to suffer from CHD (OR, 6.368; $95 \%$ CI 2.961 to $13.694, \mathrm{p}=0.000$ ) compared with the participants without DELCs.

The multivariate logistic regression analysis of the characteristics of the CHD and non-CHD groups

A multivariate logistic regression analysis (Forward: Conditional method) was used to identify the risk factors for CHD among the entire population and within gender subgroups. In the multivariate model involving the entire population, we included the following variables: age, gender, smoking status, TCH, TG, FBG, HDL-c, LDL-c, SBP, DBP and DELC; gender was excluded from the subgroup analysis. The results are reported as adjusted ORs and $95 \%$ CIs in table 5. Sex (OR, $0.321 ; 95 \%$ CI 0.203 to $0.508, \mathrm{p}=0.000)$ and DELCs

Table 3 Characteristics of the participants with and without CHD

\begin{tabular}{|c|c|c|c|c|}
\hline Characteristic & With CHD (n=445) & Without CHD (n=113) & $T$ or $Z$ or $\chi^{2}$ test & p Value \\
\hline Age (years) & $64.07 \pm 10.59$ & $61.89 \pm 9.51$ & -2.002 & 0.046 \\
\hline Sex (male/female) & $346 / 99$ & $56 / 57$ & 35.569 & 0.000 \\
\hline Smoking status (yes/no) & $166 / 279$ & $25 / 88$ & 9.223 & 0.002 \\
\hline Total cholesterol (mmol/L) & 4.07 (3.43 to 4.85$)$ & 3.97 (3.25 to 4.77$)$ & -0.979 & 0.328 \\
\hline Triglycerides $(\mathrm{mmol} / \mathrm{L})$ & $1.45(1.12$ to 2.08$)$ & $1.56(1.09$ to 2.17$)$ & -0.608 & 0.543 \\
\hline Glucose (mmol/L) & 5.28 (4.78 to 6.29$)$ & 5.14 (4.63 to 5.73$)$ & -2.211 & 0.027 \\
\hline HDL-c (mmol/L) & 0.99 (0.85 to 1.17$)$ & 1.02 (0.88 to 1.25$)$ & -1.579 & 0.114 \\
\hline LDL-c (mmol/L) & $2.62 \pm 0.81$ & $2.47 \pm 0.75$ & -1.836 & 0.067 \\
\hline $\mathrm{SBP}(\mathrm{mm} \mathrm{Hg})$ & 130 (120 to 143$)$ & 125 (120 to 138$)$ & -2.008 & 0.045 \\
\hline $\mathrm{DBP}(\mathrm{mm} \mathrm{Hg})$ & $76(70$ to 84$)$ & $75(70$ to 80$)$ & -0.759 & 0.448 \\
\hline $\operatorname{DELC}(0 / 1 / 2)$ & $100 / 77 / 268$ & $69 / 14 / 30$ & 64.781 & 0.000 \\
\hline DELC (no/yes) & $100 / 345$ & $69 / 44$ & 63.560 & 0.000 \\
\hline Gensini scores & 36.00 (20.00 to 68.00$)$ & $2.00(0.00$ to 3.00$)$ & -15.818 & 0.000 \\
\hline
\end{tabular}




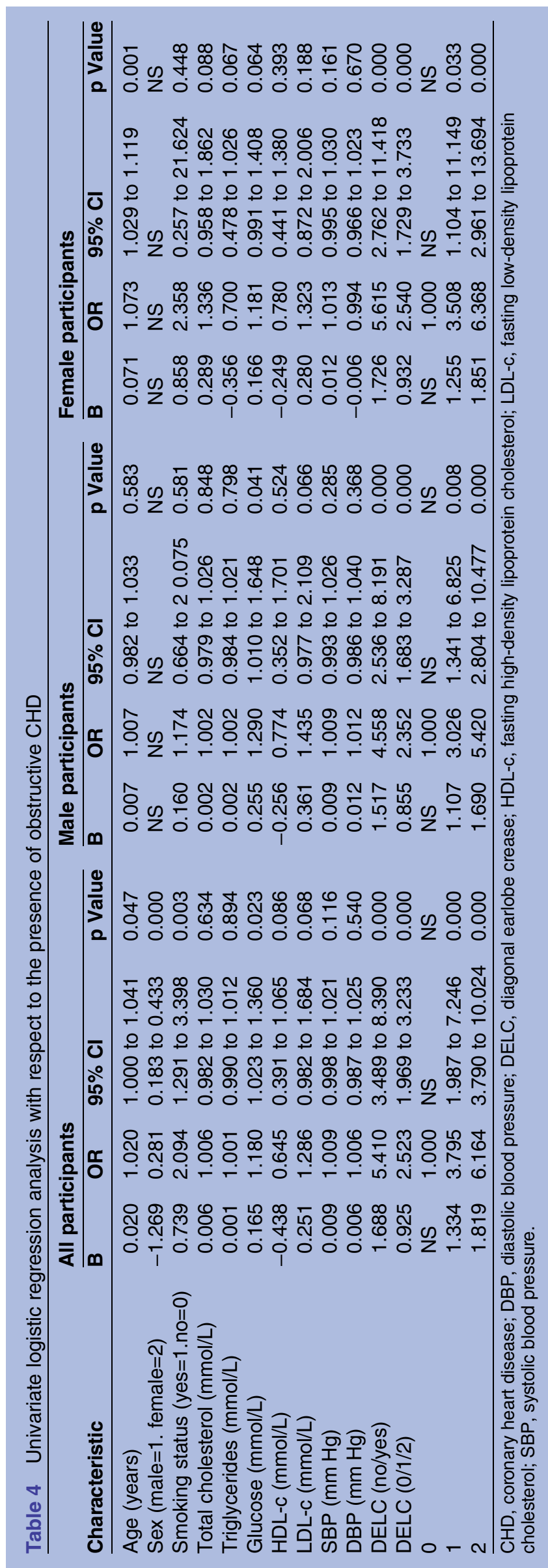

(OR, 4.861; 95\% CI 3.093 to 7.642, $\mathrm{p}=0.000$ ) remained independently associated with the risk of CHD among the entire population. The results of the subgroup analysis indicated that the risk of suffering from CHD increased if a DELC was present, in males (OR, 4.529; $95 \%$ CI 2.516 to $8.153, \mathrm{p}=0.000$ ) and in females (OR, 5.936; $95 \%$ CI 2.847 to $12.378, \mathrm{p}=0.000$ ).

It was assumed that participants without DELCs have a certain background risk of CHD (OR is assumed to be 1); the results of the multivariate logistic regression analysis (Forward: Conditional method) indicated that the relative risk of CHD among the participants with a unilateral DELC was 3.113 among all participants (OR, 3.113; 95\% CI 1.597 to $6.067, \mathrm{p}=0.001$ ), 2.918 among the male participants (OR, 2.918; 95\% CI 1.289 to $6.604, \mathrm{p}=0.010)$ and 3.178 among the female participants $(\mathrm{OR}, 3.178 ; 95 \%$ CI 0.990 to $10.207, \mathrm{p}=0.052$ ). The relative risk of $\mathrm{CHD}$ among the participants with bilateral DELCs was 5.690 among all participants (OR, 5.690; 95\% CI 3.450 to 9.384, $\mathrm{p}=0.000), 5.436$ among the male participants (OR, $5.436 ; 95 \%$ CI 2.808 to $10.523, \mathrm{p}=0.000)$ and 7.148 among the female participants (OR, 7.148; 95\% CI 3.184 to $16.049, \mathrm{p}=0.000$ ).

\section{The relationship between DELC and classical risk factors}

The analysis of the possible relationships between the DELCs and the classical risk factors is shown in table 6 . Given that the conventional risk of participants unexposed to both classical risk factors and DELCs (reference category) was 1.0 , the OR estimating the effect of exposure to both DELC and age (older than 60 years of age), sex (male gender) and smoking status, was significantly higher compared with the ORs estimating the effect of each factor in the absence of the other. The proportion of CHD attributable to the interaction of DELC and age was $13 \%(\mathrm{SI}=1.21, \mathrm{SIM}=1.65, \mathrm{AP}=0.132)$, for DELC and sex it was $49 \% \quad(\mathrm{SI}=2.09, \mathrm{SIM}=0.81$, $\mathrm{AP}=0.49$ ), while for DELC and smoking status it was $29 \%$ (SI=1.49, $\mathrm{SIM}=0.73, \mathrm{AP}=0.29$ ).

\section{An ROC analysis of the DELCs with respect to the detection of CHD}

An ROC analysis of the DELCs with respect to the detection of CHD is depicted in figure 7; the sensitivity of the sign was $78 \%$; the specificity was $61 \%$; the positive predictive value was $89 \%$; the negative predictive value was $41 \%$, and the Youden index was 0.39 with respect to the diagnosis of CHD in this hospital-based population (area under the curve $0.693,95 \%$ CI 0.636 to 0.750 , $\mathrm{p}=0.000)$.

\section{DISCUSSION}

In this hospital-based cross-sectional study of adult Chinese participants with angiographic evidence of coronary atherosclerosis, the primary findings were as follows: DELCs were independently and significantly associated with an increased prevalence of $\mathrm{CHD}$, as 
Table 5 A multivariate logistic regression analysis with respect to the presence of obstructive CHD

\begin{tabular}{lllll}
\hline Characteristic & B-coefficient & OR & 95\% Cl & p Value \\
\hline All participants & & & & \\
Sex (male=1. female=2) & -1.136 & 0.321 & 0.203 to 0.508 & 0.000 \\
DELC (yes/no) & 1.581 & 4.861 & 3.093 to 7.642 & 0.000 \\
Sex (male=1.female=2) & -1.175 & 0.309 & 0.195 to 0.489 & 0.000 \\
DELC (0/1/2) & 0.878 & 2.406 & 1.868 to 3.097 & 0.000 \\
0 & NS & 1.000 & NS & NS \\
1 & 1.136 & 3.113 & 1.597 to 6.067 & 0.001 \\
2 & 1.739 & 5.690 & 3.450 to 9.384 & 0.000 \\
Male participants & & & & 0.000 \\
DELC (yes/no) & 1.511 & 4.529 & 2.516 to 8.153 & 0.000 \\
DELC (0/1/2) & 0.855 & 2.351 & 1.683 to 3.285 & NS \\
0 & $\mathrm{NS}$ & 1.000 & $\mathrm{NS}$ & 0.010 \\
1 & 1.071 & 2.918 & 1.289 to 6.604 & 0.000 \\
2 & 1.693 & 5.436 & 2.808 to 10.523 & 0.000 \\
Female participants & & & & 0.000 \\
DELC (yes/no) & 1.781 & 5.936 & 2.847 to 12.378 & NS \\
DELC (0/1/2) & 0.989 & 2.689 & 1.794 to 4.030 & 0.052 \\
0 & $\mathrm{NS}$ & 1.000 & $\mathrm{NS}$ & 0.000 \\
1 & 1.156 & 3.178 & 0.990 to 10.207 & \\
2 & 1.967 & 7.148 & 3.184 to 16.049 & \\
\hline
\end{tabular}

$\mathrm{CHD}$, coronary heart disease; DBP, diastolic blood pressure; DELC, diagonal earlobe crease; HDL-c, fasting high-density lipoprotein cholesterol; LDL-c, fasting low-density lipoprotein cholesterol; SBP, systolic blood pressure.

estimated via coronary angiography, and the positive correlations between DELC and age, gender and smoking status correlated significantly with the risk of $\mathrm{CHD}$; nevertheless, the relative risk of CHD among the participants with bilateral DELCs was the highest among the participants without DELCs, the participants with a unilateral DELC, and the participants with bilateral DELCs.

Atherosclerosis is a major source of disability and death in China; as China has grown economically, it has experienced an epidemiological transition with respect to mortality secondary to ischaemic heart disease, as said mortality has more than doubled within the past two decades, and now exceeds 1 million deaths per year. ${ }^{15}$ Many of these adverse events took place in individuals free of prodromal symptoms, many of whom were without classical risk factors such as hypertension, hyperlipidaemia, diabetes or obesity. This 'detection gap' has spurred clinicians to attempt to identify extravascular

Table 6 Relationship between DELCs and the classical risk factors

\begin{tabular}{|c|c|c|c|c|c|}
\hline Classical risk & DELC & CHD & Controls & OR $(95 \% \mathrm{Cl})$ & p Value \\
\hline \multicolumn{6}{|l|}{ Age } \\
\hline 0 & 0 & 59 & 33 & 1 & 0.000 \\
\hline 0 & 1 & 83 & 11 & $4.220(1.974$ to 9.021$)$ & 0.000 \\
\hline 1 & 0 & 41 & 36 & $0.637(0.343$ to 1.182$)$ & 0.153 \\
\hline \multirow[t]{2}{*}{1} & 1 & 262 & 33 & 4.441 (2.539 to 7.767$)$ & 0.000 \\
\hline & $\mathrm{SI}=1.21$ & $\mathrm{SIM}=1.65$ & $\mathrm{RERI}=0.58$ & $\mathrm{AP}=0.132$ & \\
\hline 0 & 1 & 73 & 19 & 5.615 (2.762 to 11.418$)$ & 0.000 \\
\hline 1 & 0 & 74 & 31 & 3.489 (1.818 to 6.694$)$ & 0.000 \\
\hline \multirow[t]{2}{*}{1} & 1 & 272 & 25 & 15.902 (8.339 to 30.322$)$ & 0.000 \\
\hline & $\mathrm{SI}=2.09$ & $\mathrm{SIM}=0.81$ & RERI=7.79 & $\mathrm{AP}=0.49$ & \\
\hline \multicolumn{6}{|l|}{ Smoking status } \\
\hline 0 & 0 & 62 & 55 & 1 & 0.000 \\
\hline 1 & $\mathrm{SI}=1.49$ & $\mathrm{SIM}=0.73$ & $\mathrm{RERI}=3.08$ & $\mathrm{AP}=0.29$ & \\
\hline
\end{tabular}




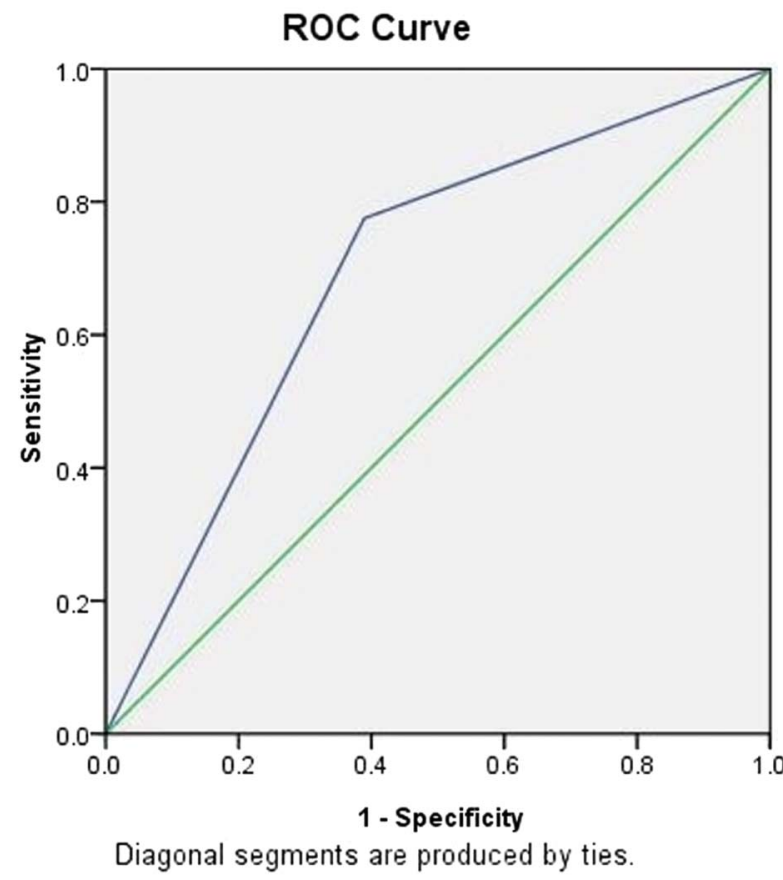

Figure 7 The receiver operating characteristic curve of DELC with respect to the diagnosis of CHD. CHD, coronary heart disease; DELC, diagonal earlobe crease.

physical signs that can identify individuals at high risk of atherosclerosis. ${ }^{6}$ Preliminary uncontrolled observations by a pulmonologist that a 'positive ear-lobe sign' is associated with the development of premature coronary artery atherosclerosis have been heralded. ${ }^{5}$ The prognostic significance of DELCs has subsequently been substantiated in more than 40 separate studies, as reviewed by Friedlande ${ }^{6}$ which may reflect differences in age groups, the extent of CHD, ethnic distributions, the criteria for the diagnosis of CHD (based on the presence of chest pain, risk factors, electrocardiographic abnormalities at rest, or CAG) and criteria for the diagnosis of DELCs (either unilateral or bilateral; length, depth or inclination.) However, the relationship between DELCs and CHD remains controversial due to the confounding effects of the above variables. One study evaluating the association between the presence of diagonal earlobe creases and coronary artery disease has shown that the presence of DELCs was related to CHD and the extent of CHD as measured by the number of major arteries narrowed. However, the difference for the recruited criteria of the case and control was the major limitation of the study, because 1086 consecutive patients who denied symptoms of myocardial ischaemia and were admitted to a general hospital for other reasons were recruited as controls, and 338 patients with documented CHD via angiography were employed as cases. ${ }^{16}$ Another study demonstrated that DELC was independently and significantly associated with increased prevalence, extent and severity of CHD, and the cases and controls were defined as the result of coronary CT angiography. ${ }^{17}$ In addition, the relationship between the DELC and CHD has been confirmed in hospital-based participants ${ }^{8}$ and community-based general population, ${ }^{18}$ however, the criteria for the diagnosis of CHD are based on the presence of chest pain, risk factors, ECG and other medical histories rather than coronary angiography, and this should be taken into account when interpreting the above results to the general population. In the present study, the sample is comprised solely of Han Chinese people; CHD and control were diagnosed through angiography, and DELC status was strictly defined as without or with unilateral or bilateral DELCs. The primary finding of the present study was that DELCs were significantly linked with CHD, independent of classical cardiovascular risk factors; this study also demonstrated that participants with bilateral DELCs exhibited more stenotic vessels and a higher prevalence of significant coronary artery stenosis compared with the participants without DELCs, as well as those with unilateral DELCs. To the best of our knowledge, this is the first study to investigate the relationship between unilateral DELCs, bilateral DELCs and CAG-certified CHD.

In the present study, the participants with bilateral DELCs were more frequently male and were significantly older. Although smoking status was a strong risk factor for CHD, we observed no differences in the prevalence of either parameter with respect to DELC. In order to analyse the positive and negative correlations between DELC and the classical risk factors, a $4 \times 2$ table was used; the synergy measures in additive (SI) models of DELC with age, male gender and smoking status were 1.21, 2.09 and 1.49, respectively. Therefore, positive correlations between DELC and age, male gender and smoking status were observed, and the proportions of CHD attributable to the DELCs and age, male gender and smoking status were approximately $13 \%, 49 \%$ and $29 \%$, respectively. The relationship between DELC and the classical risk factors with respect to the severity and the prevalence of CHD has not been reported previously; therefore, the results of the present study may shed light on the relationship between CHD and DELCs.

The pathophysiological mechanism underlying the correlation between DELCs and CHD remains unclear. Some suggested mechanisms include the following: as many traits reflective of microvascular disease were identified in biopsy specimens taken from the earlobes and the coronary bed, the unbalanced ratio of collagen to elastin and the degeneration of elastin may partially account for the relationship between CHD and DELC; $;^{19}$ not only are DELCs rare in infants, but skin ageing also parallels the ageing process of the coronary arteries; therefore, DELCs may reflect skin ageing; ${ }^{20}$ additionally, a study of Japanese male participants with DELCs and metabolic syndrome demonstrated shortened telomeres (extreme ends of chromosomal DNA) in the participants' peripheral white blood cells, which also implicates ageing. ${ }^{21}$ Given that earlobe collagen consists of peptide chains resembling those found on scavenger macrophage receptors used for the ingestion of atheromatous 
cholesterol, DELCs and CAD may share a common pathophysiology. ${ }^{22}$ However, the exact mechanism underlying the relationship between CHD and DELC warrants further study.

The strength of this study is that it used coronary angiography and a standard evaluation sheet for the diagnosis of CHD and DELC, respectively. Further, the present study explores the differentiation of unilateral versus bilateral DELC for the diagnosis of CHD. However, this study had several limitations. Its sample size was small; large-scale studies are necessary to substantiate our findings. Furthermore, given that the study involved patients hospitalised for catheterisation with either known or suspected coronary artery disease, its results may not be applicable to an unselected community population, besides, the ratio case/control regarding CHD or the ratio male/female seems unbalance; therefore, community-based large-scale cohort studies are necessary to substantiate our results. Additionally, the pathogenesis of the apparent relationship between DELC and CHD was not studied; therefore, although a statistical relationship appears to exist, the biological plausibility of said relationship remains unproven and warrants clarification in future studies.

The present study suggested that bilateral DELCs are both a simple and a feasible method of identifying CHD. The exact mechanism underlying this relationship warrants further study, however.

\section{Author affiliations}

${ }^{1}$ Department of Cardiovascular Medicine, The First Affiliated Hospital of Nanjing Medical University, Nanjing, Jiangsu Province, China ${ }^{2}$ Department of Cardiovascular Medicine, The People's Hospital of Gaochun, Nanjing, Jiangsu Province, China

${ }^{3}$ Department of Cardiovascular Medicine, The Jurong City Hospital of Traditional Chinese Medicine, Zhenjiang, Jiangsu Province, China

Contributors As the guarantor, EZJ conceived the study. YW drafted the initial paper. L-HM and Z-YL enrolled participants and collected the data under the supervision of T-BZ and C-JL, while X-QD, P-CG and ZL cleaned and analysed the data. Z-JY, W-ZM and L-SW monitored the conduct of the study, and reviewed the safety and effectiveness of the data. All the authors reviewed the manuscript.

Funding This study was supported by the National Natural Science Foundations of China (grants 81170180, 30400173 and 30971257), a project funded by the Priority Academic Program Development of Jiangsu Higher Education Institutions. E-ZJ is an Assistant Fellow at the Collaborative Innovation Center for Cardiovascular Disease Translational Medicine.

Competing interests None declared.

Patient consent Obtained.

Ethics approval Ethics Committee of the First Affiliated Hospital of Nanjing Medical University.

Provenance and peer review Not commissioned; externally peer reviewed.

Data sharing statement No additional data are available.
Open Access This is an Open Access article distributed in accordance with the Creative Commons Attribution Non Commercial (CC BY-NC 4.0) license, which permits others to distribute, remix, adapt, build upon this work noncommercially, and license their derivative works on different terms, provided the original work is properly cited and the use is non-commercial. See: http:// creativecommons.org/licenses/by-nc/4.0/

\section{REFERENCES}

1. Nowbar AN, Howard JP, Finegold JA, et al. 2014 global geographic analysis of mortality from ischaemic heart disease by country, age and income: statistics from World Health Organisation and United Nations. Int J Cardiol 2014;174:293-8.

2. Wu X, Yang D, Zhao Y, et al. Effectiveness of percutaneous coronary intervention within 12 hours to 28 days of ST-elevation myocardial infarction in a real-world Chinese population. PLOS ONE 2013;8:e58382.

3. Wu XL, Yang DY, Zhao YS, et al. Diagonal earlobe crease and coronary artery disease in a Chinese population. BMC Cardiovasc Disord 2014;14:43.

4. Yin Huihe. The basic theory of Chinese traditional medicine. 2nd edn. Beijing: People's Medical Publishing House Co.,Ltd (PMPH), 1985:9.

5. Frank ST. Aural sign of coronary-artery disease. $N$ Engl J Med 1973;289:327-8.

6. Friedlande AH, López-López J, Velasco-Ortega E. Diagonal ear lobe crease and atherosclerosis: a review of the medical literature and dental implications. Med Oral Patol Oral Cir Bucal 2012;17:e153-9.

7. Elliott WJ, Powell LH. Diagonal earlobe creases and prognosis in patients with suspected coronary artery disease. Am J Med 1996;100:205-11.

8. Rodríguez-López C, Garlito-Díaz H, Madroñero-Mariscal R, et al. Earlobe crease shapes and cardiovascular events. Am J Cardiol 2015;116:286-93.

9. Judkins MP. Selective coronary arteriography. I. A percutaneous transfemoral technic. Radiology 1967:89:815-24.

10. Sun $X$, Zhang $M$, Sanagawa A, et al. Circulating microRNA-126 in patients with coronary artery disease: correlation with LDL cholesterol. Thromb J. 2012;10:16

11. Gensini GG. A more meaningful scoring system for determinating the severity of coronary heart disease. Am J Cardiol 1983;51:606.

12. Shrestha I, Ohtsuki T, Takahashi T, et al. Diagonal ear-lobe crease is correlated with atherosclerotic changes in carotid arteries. Circ $J$ 2009;73:1945-9.

13. Yang Q, Khoury MJ. Evolving methods in genetic epidemiology. III. Gene-environment interaction in epidemiologic research. Epidemiol Rev 1997; 19:33-43.

14. Hanley JA, McNeil BJ. The meaning and use of the area under a receiver operating characteristic (ROC) curve. Radiology 1982;143:29-36.

15. Yang G, Wang $Y$, Zeng $Y$, et al. Rapid health transition in China, 1990-2010: findings from the Global Burden of Disease Study 2010 Lancet 2013;381:1987-2015.

16. Tranchesi Júnior $B$, Barbosa $V$, de Albuquerque $C P$, et al. Diagonal earlobe crease as a marker of the presence and extent of coronary atherosclerosis. Am J Cardiol 1992;70:1417-20.

17. Shmilovich H, Cheng VY, Rajani R, et al. Relation of diagonal ear lobe crease to the presence, extent, and severity of coronary artery disease determined by coronary computed tomography angiography. Am J Cardiol 2012;109:1283-7.

18. Christoffersen M, Frikke-Schmidt $\mathrm{R}$, Schnohr $\mathrm{P}$, et al. Visible age-related signs and risk of ischemic heart disease in the general population: a prospective cohort study. Circulation 2014;129:990-8.

19. Kaukola S. The diagonal ear-lobe crease, a physical sign associated with coronary heart disease. Acta Med Scand Suppl 1978;619:1-49.

20. Bouissou H, Pieraggi MT, Julian M, et al. [Value of skin biopsy in coronary insufficiency]. Arch Mal Coeur Vaiss 1973;66:655-60.

21. Higuchi Y, Maeda T, Guan JZ, et al. Diagonal earlobe crease are associated with shorter telomere in Male Japanese patients with metabolic syndrome. Circ J 2009;73:274-9.

22. Sapira JD. Earlobe creases and macrophage receptors. South Med J 1991;84:537-8. 